\title{
Prevalence and Treatment Outcomes of Marine-Lenhart Syndrome in Japan
}

\author{
Hirosuke Danno ${ }^{a}$ Eijun Nishihara ${ }^{a}$ Kazuyoshi Kousaka ${ }^{a}$ Tomohiko Nakamura ${ }^{a}$ \\ Toshihiko Kasahara ${ }^{a}$ Takumi Kudo $^{a}$ Mitsuru Ito $^{a}$ Shuji Fukata ${ }^{a}$ \\ Mitsushige Nishikawa ${ }^{a}$ Akira Miyauchib \\ aDivision of Internal Medicine, Center for Excellence in Thyroid Care, Kuma Hospital, Kobe, Japan; ${ }^{b}$ Division of \\ Surgery and Director, Center for Excellence in Thyroid Care, Kuma Hospital, Kobe, Japan
}

\section{Keywords}

Graves' disease · Hyperthyroidism • Marine-Lenhart syndrome $\cdot$ Thyroid nodule $\cdot$ Toxic adenoma

\begin{abstract}
Introduction: Marine-Lenhart syndrome (MLS) is now understood to be a combination of Graves' disease and autonomously functioning thyroid nodule(s) (AFTNs). The prevalence of the syndrome and suitable treatments for those living in iodine-sufficient areas are uncertain. Objectives: We aimed to investigate the prevalence, treatment, and prognosis of MLS in Japan, an iodine-sufficient area. Methods: This study involved patients who visited our hospital between February 2005 and August 2019. Among patients with both thyrotoxicosis and thyroid nodule(s) larger than $10 \mathrm{~mm}$, MLS and isolated AFTNs were diagnosed based on serum thyroidstimulating hormone receptor antibody levels and scintigraphy using radioiodine or technetium-99m and thyroid uptake. Results: Twenty-two patients were found to have MLS, compared to 372 with isolated AFTNs and 8,343 with Graves' disease, during the period. Therefore, the rate of MLS cases was $0.26 \%$ among all patients with Graves' disease $(22 / 8,343)$. Treatments and outcomes were assessed for cases of MLS ( $n$ $=18$ ) and isolated AFTNs $(n=269)$. Antithyroid drugs (ATDs) were withdrawn in $27.8 \%$ of cases in the MLS group and
\end{abstract}

$10.3 \%$ in the isolated AFTN group. There was no significant difference in the clinical outcome after ATD withdrawal between the 2 groups. However, the rate of hypothyroidism after radioactive iodine (RAI) administration was significantly higher in the MLS group than in the isolated AFTN group (42.9 vs. $9.0 \%, p=0.005)$ despite similar doses of RAl. Conclusions: The prevalence of MLS among patients with Graves' disease was $0.26 \%$ in Japan. RAI therapy induces hypothyroidism more frequently than in those with AFTNs probably because RAl is taken up in the surrounding Graves' tissues.

(c) 2020 The Author(s)

Published by S. Karger AG, Basel

\section{Introduction}

The Marine-Lenhart syndrome (MLS), first described by Charkes in 1972 [1], is now commonly defined as "a combination of Graves' disease and autonomous functioning thyroid nodule(s) (AFTN)" [2-4]. Typical scintigraphic images include both increased uptake in the affected nodule(s) and diffuse uptake in the extranodular tissue. In contrast, AFTN (solitary toxic adenoma or toxic multinodular goitre) is defined as single or scattered hot nodule(s) with suppressed extranodular tissue.
(C) 2020 The Author(s)

Published by S. Karger AG, Basel

This is an Open Access article licensed under the Creative Commons Attribution-NonCommercial-4.0 International License (CC BY-NC) (http://www.karger.com/Services/OpenAccessLicense), applicable to the online version of the article only. Usage and distribution for commercial purposes requires written permission.
Hirosuke Danno

Center for Excellence in Thyroid Care

Kuma Hospital, 8-2-35, Shimoyamate-Dori, Chuo-Ku

Kobe-City Hyogo 650-0011 (Japan)

danhirosuke@kuma-h.or.jp 
AFTNs are common in iodine-deficient areas, accounting for up to $60 \%$ of cases of thyrotoxicosis. However, it is rare $(3-10 \%$ of cases of thyrotoxicosis) in regions with sufficient iodine supply [5]. The prevalence of AFTNs in iodine-sufficient areas is unclear.

Regarding treatment of MLS, radioactive iodine (RAI) comprising 13-26 $\mathrm{mCi}$ and 13-40 $\mathrm{mCi}$ resulted in euthyroidism [6-8] and hypothyroidism [6,8-10], respectively. Some cases of thyroidectomy (Tx) have been reported $[2,11-18]$, and others showed normalization of thyroid function with antithyroid drugs (ATDs) [2, 6, 7, 19]. However, none of these reports were conclusive regarding the most suitable treatment partly due to the short observation periods after treatment. To investigate the clinical significance of MLS, we assessed the frequency, treatment, and prognosis of the syndrome at a hospital with thyroid specialists in Japan, an iodine-sufficient country with an average daily iodine intake of $1.2 \mathrm{mg}$ [20].

\section{Materials and Methods}

\section{Patients}

This study involved patients who visited our hospital between February 2005 and August 2019. Thyrotoxicosis was diagnosed when the thyroid-stimulating hormone (TSH) concentration was $\leq 0.1 \mu \mathrm{U} / \mathrm{mL}$, and FT4 and/or FT3 level was high. Graves' disease was diagnosed based on the following criteria: (i) thyrotoxicosis, (ii) positive TSH receptor antibody (TRAb), and (iii) non-suppressed radioiodine or technetium-99m thyroid uptake [21]. AFTN (solitary toxic adenoma or toxic multinodular goitre) was diagnosed when patients had both thyrotoxicosis and nodule(s) $>10 \mathrm{~mm}$ in diameter and for which the radioisotope accumulated (hot nodule). MLS was diagnosed when a combination of Graves' disease and AFTN was confirmed. Treatments and outcomes of patients with MLS and AFTN only were assessed retrospectively by follow-up for $>12$ months after treatment.

\section{Laboratory Methods}

Between February 2005 and December 2018, serum TSH, FT4, and FT3 levels were measured using chemiluminescence immunoassays with Architect kits (Abbott, Japan) (normal ranges of TSH, 0.3-5.0 $\mu \mathrm{U} / \mathrm{mL}$; FT4, 0.7-1.6 ng/dL; and FT3, 1.7-3.7 pg/mL). After January 2019, serum levels of these markers were measured using electrochemiluminescence immunoassays with ECLusys kits (Roche Diagnostics, Japan) (normal ranges of TSH, 0.5-5.0 $\mu \mathrm{U} /$ $\mathrm{mL}$; FT4, 0.9-1.7 ng/dL; and FT3, 2.3-4.0 pg/mL). Serum TRAb levels were measured via ELISA using TRAb-ELISA (Cosmic Corp., Tokyo, Japan) (normal range $<15 \%$ ) before July 2008 and via electrochemiluminescence immunoassays using ECLusys kits (Roche Diagnostics, Japan) (normal range $<2.0$ IU/L) after August 2008. Thyroid scintigraphy (planar image) was performed using radioiodine in 1,026 patients $(81.9 \%$ ) and technetium (Tc)- $99 \mathrm{~m}$ in 227 patients $(18.1 \%)$ as tracers. The normal lower limits of radioiodine uptake were $5 \%$ for $3 \mathrm{~h}$ and $10 \%$ for $24 \mathrm{~h}$ and that of Tc- $99 \mathrm{~m}$ uptake was $0.5 \%$ for $20 \mathrm{~min}$. Ultrasound examination was per- formed by well-trained registered medical sonographers using TOSHIBA Aplio SSA-700A or SSA-770A systems with PLT805AT $(8 \mathrm{MHz})$ and PLT-1204AX (12 MHz) linear probes between February 2005 and July 2012, and TOSHIBA Aplio TUSA500 (versions 3.0, 5.0, and 6.0) systems with PLT-805AT (8 MHz) and PLT-1005BT (10 MHz) linear probes after August 2012.

\section{Treatment Protocol}

ATD, RI, and Tx were performed as treatments. Methimazole was the first choice for ATD, with an initial dose of 10-30 mg depending on the FT4 level. The starting dose was gradually reduced as thyrotoxicosis improved [21]. Methimazole was switched to propylthiouracil or potassium iodide when adverse reactions occurred. The initial dose of radioiodine- 131 was based on $13 \mathrm{mCi}$ and determined by each physician with reference to thyroid size and function. If hyperthyroidism continued after a single dose of radioiodine, additional doses were given. Total Tx, lobectomy, and lobectomy with isthmectomy were performed as surgical techniques.

\section{Statistical Analysis}

The Mann-Whitney U test was used to compare ages, RAI doses, and nodular sizes between the MLS and AFTN groups. Pearson's $\chi^{2}$ test was used to compare sex differences. Bonferroni methods were used to adjust for multiple testing. The contingency table was analysed using Fisher's exact test (in less than 20 cases) and Pearson's $\chi^{2}$ test (in equal or more than 20 cases) for independence and residual analysis. Analysis items with a two-sided $p<0.05$ were considered statistically significant. Statistical analyses were performed using StatFlex version 6.0 (Artech Co. Ltd., Tokyo, Japan).

\section{Results}

\section{Frequency Analysis}

Of 202,778 new outpatients during the study period, 25,913 patients were diagnosed with thyrotoxicosis and underwent ultrasonography. Of these, 1,253 patients had one or more nodule(s) larger than $10 \mathrm{~mm}$ and were further evaluated by TRAb levels and scintigraphy (Fig. 1). Of 579 patients with positive TRAb test results, 22 patients had hot nodule(s) and non-suppressed uptake of the extranodular thyroid gland. These patients were diagnosed with Graves' disease with AFTN, or MLS. The isolated AFTN group consisted of 372 patients with negative TRAb test results and hot nodule(s) (Fig. 1). Representative scintigraphic images are shown in Figure 2. The total number of patients with Graves' disease was 8,343 . Thus, the proportion of all patients with Graves' disease with MLS was $0.26 \%(22 / 8,343)$. In addition, the proportion of the patients with MLS to those with thyrotoxicosis with nodule(s) was $1.8 \%(22 / 1,253)$, and that of MLS to Graves' disease with nodule(s) was 3.8\% (22/[22 + 557]) (Fig. 1).

The median age was 57 (range 20-81) years in the MLS group and 53 (range 13-89) years in the AFTN group; 


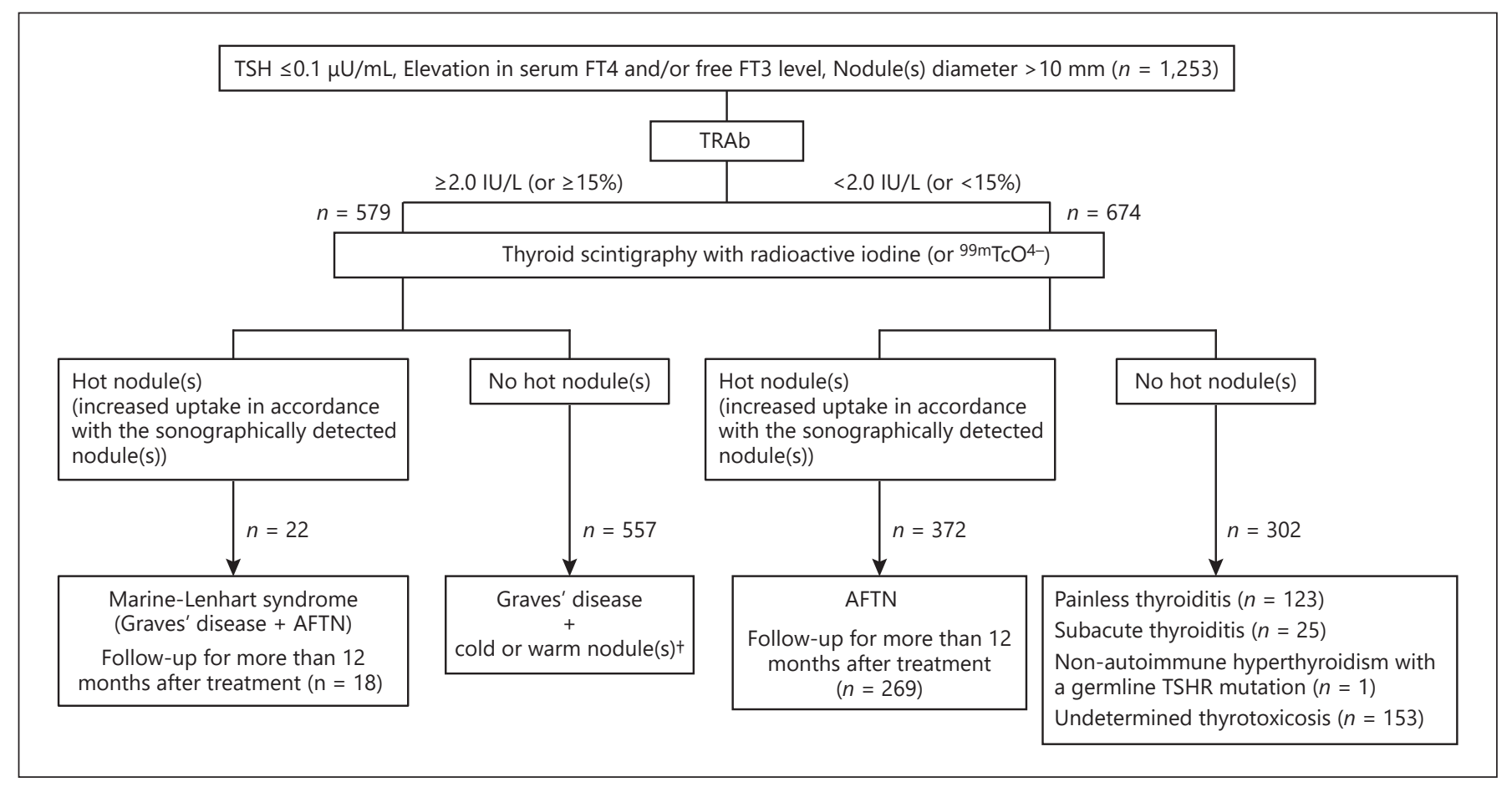

Fig. 1. Study flowchart of the identification of patients with MLS (Graves' disease + AFTN) and isolated AFTN. $\dagger$ No patients showed positive TRAb with suppressed thyroid uptake or negative TRAb with non-suppressed thyroid uptake. AFTN, autonomously functioning thyroid nodule; TRAb, TSH receptor antibody; MLS, MarineLenhart syndrome; TSH, thyroid-stimulating hormone.

there was no significant difference between the 2 groups $(p=0.23)$. Women comprised $86.4 \%$ of patients in the MLS group and $90.8 \%$ in the AFTN group; there was no significant difference between the 2 groups $(p=0.48)$.

\section{Treatment}

Treatments and outcomes were analysed among those with MLS $(n=18)$ and AFTN $(n=269)$ who were followed up for more than 12 months after treatment (Table 1). ATD constituted a significantly higher proportion at $44.1 \%(8 / 18)$ in the MLS group than in the AFTN group $(10.8 \%$ [29/269], $p<0.001)$. In contrast, the rates of RAI were similar $(p=0.596)$, but Tx constituted a significantly higher proportion at $43.9 \%(118 / 269)$ in the AFTN group than in the MLS group (16.7\% [3/18], $p=0.024)$.

The maximum diameter of nodule(s) was significantly smaller in the MLS group than in the AFTN group (median 27.0 vs. $38.0 \mathrm{~mm}, p<0.01$ ). In the AFTN group, the mean maximum diameter of the nodule(s) was larger in the Tx group than in the ATD and RAI groups (median 41.0 vs. $31.6 \mathrm{~mm}, p<0.001$ and 41.0 vs. $34.0 \mathrm{~mm}, p<$ 0.001 , respectively). Although not significantly different, a similar trend was observed in the MLS group (55.0 vs. $23.0 \mathrm{~mm}, p=0.09$ and 55.0 vs. $31.0 \mathrm{~mm}, p=0.072$, respectively). There was no statistically significant difference in the maximum diameter of the nodule(s) between the MLS and AFTN groups or in the RAI and Tx groups, but the ATD group had significantly smaller nodule(s) than did the MLS group ( 23.0 vs. $31.6 \mathrm{~mm}, p=0.027$ ).

Table 2 shows the distribution of thyroid function after ATD withdrawal in the MLS and AFTN groups. ATDs were withdrawn in 5 of 18 patients $(27.8 \%)$ in the MLS group and 3 of 29 (10.3\%) patients in the AFTN group, with no significant difference between the 2 groups $(p=$ 0.23 ). All patients presented with euthyroidism, and all patients in the MLS group were TRAb negative before ATD withdrawal. Subclinical hyperthyroidism (low TSH levels with normal FT4 and FT3 levels) occurred in 2 patients in the MLS group and 2 in the AFTN group after ATD withdrawal. Three patients in the MLS group and one in the AFTN group remained euthyroid after ATD withdrawal. There was no significant difference in clinical outcomes between the 2 groups $(p=0.571)$. 
Fig. 2. Representative scintigraphic images of Graves' disease, MLS, Graves' disease + cold nodule, and AFTN. Iodine-131 thyroid uptake at $3 \mathrm{~h}$ was $67.8 \%$ (normal $5-15 \%)$ in (a). Iodine-131 thyroid uptake at $24 \mathrm{~h}$ was $35.5 \%$ (normal $10-40 \%$ ) in (b), $21.0 \%$ in (c), and $31.1 \%$ in (d). + represents suprasternal notch. a Graves' disease: thyroid scan shows increased diffuse uptake in both thyroid lobes. b MLS: thyroid scan demonstrates a hot area in the isthmus consistent with hyperfunctioning nodule (arrowhead). The remaining tissue showed a slightly increased uptake as expected in Graves' disease. c Graves' disease + cold nodule: there is a photopenic area (arrowhead) in the right thyroid lobe with increased uptake in the remaining tissue. d AFTN (solitary toxic adenoma): thyroid scintigraphy shows a functioning hot nodule in the left lobe. No background tracer accumulates. AFTN, autonomously functioning thyroid nodule; MLS, Marine-Lenhart syndrome.

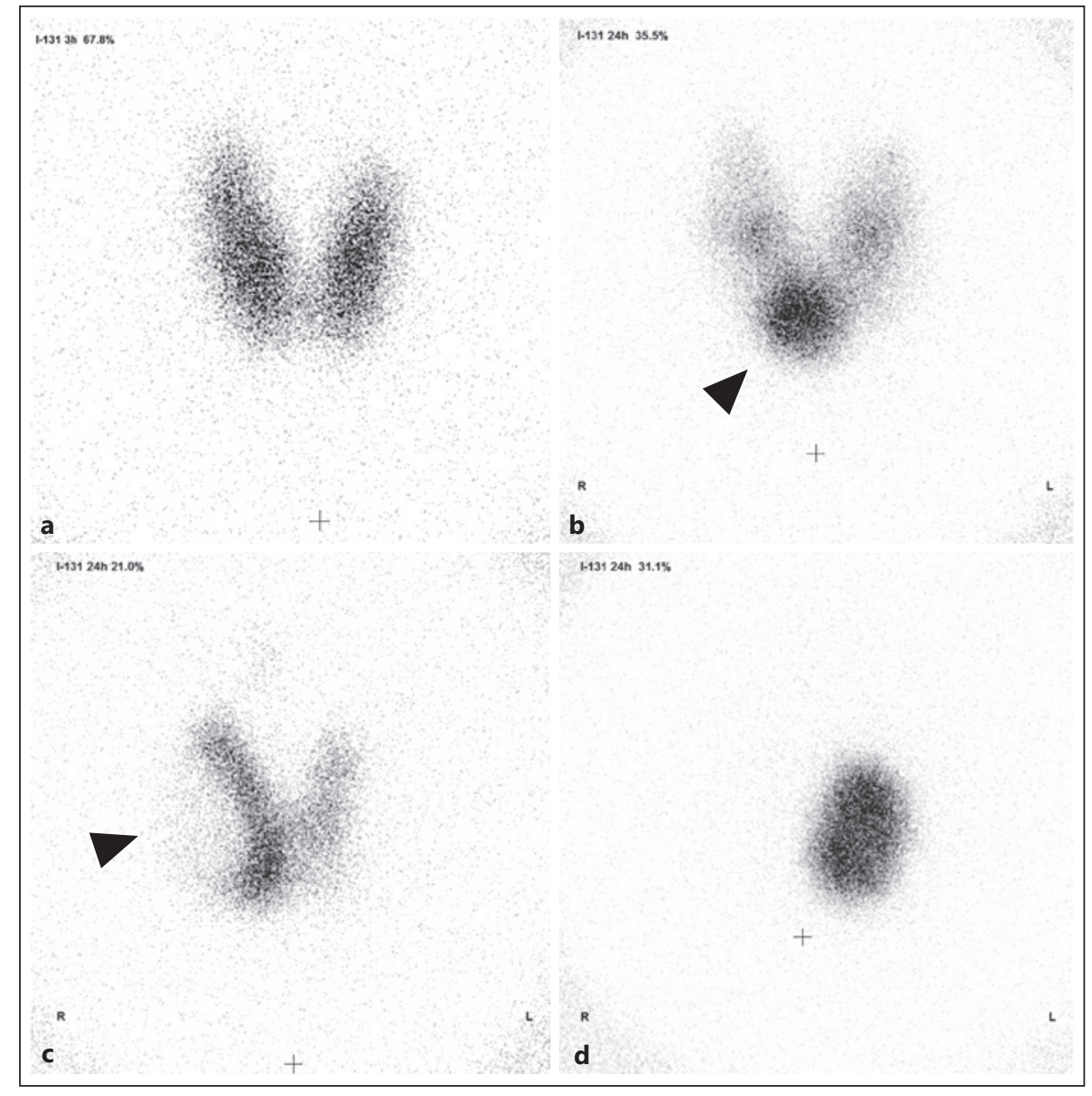

Table 1. Details of treatment (data indicate number of patients and nodule size)

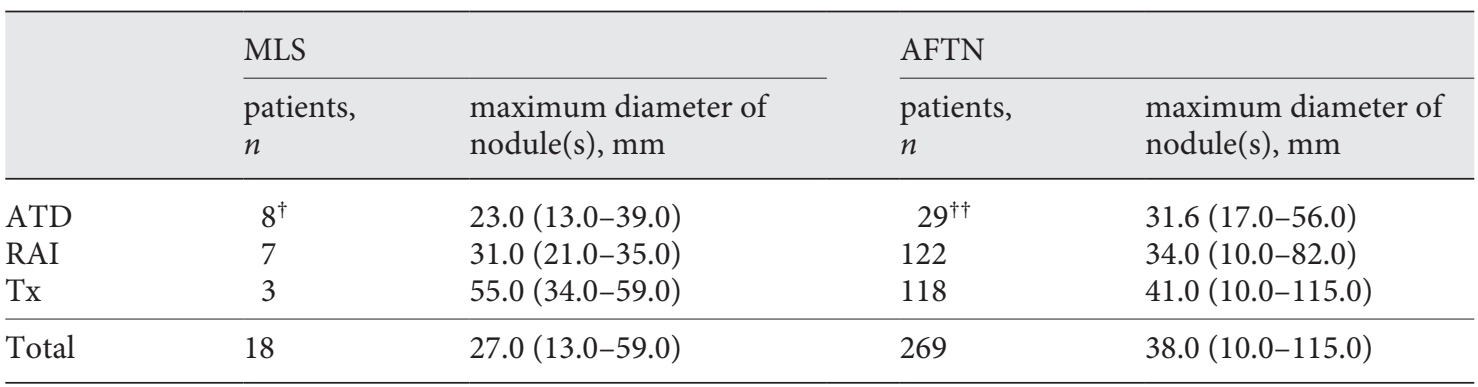

In the case of multiple autonomously functioning thyroid nodules ( 3 cases in the MLS group, 68 cases in the AFTN group), the largest nodule was measured. Data regarding nodule size are reported as medians (ranges). The surgical technique for MLS was total thyroidectomy $(n=3)$ and for AFTN, lobectomy or lobectomy with isthmectomy $(n=80)$ or total thyroidectomy $(n=38) .{ }^{\dagger}$ One patient received potassium iodide, one patient received propylthiouracil, others received methimazole. ${ }^{\dagger \dagger}$ All patients received methimazole. MLS, MarineLenhart syndrome; AFTN, autonomously functioning thyroid nodule; ATD, antithyroid drug; RAI, radioactive iodine; Tx, thyroidectomy. 
Table 2. Comparison of thyroid function after ATD withdrawal between MLS and AFTN groups

\begin{tabular}{lll}
\hline & $\begin{array}{l}\text { Subclinical hyperthyroidism } \\
\text { patients, } n \text { [duration of normal } \\
\text { thyroid function after ATD } \\
\text { withdrawal, months] }\end{array}$ & $\begin{array}{l}\text { Euthyroidism patients, } \\
n \text { [follow-up period } \\
\text { after ATD withdrawal, } \\
\text { months] }\end{array}$ \\
\hline $\operatorname{MLS}(n=5)$ & $2[2.2(1.4-3.0)]^{\dagger}$ & $3[9.9(2.5-18.1)]^{\dagger}$ \\
$\operatorname{AFTN}(n=3)$ & $2[11.4(9.5-13.3)]^{\dagger}$ & $1[46.1]$ \\
\hline
\end{tabular}

Fisher's exact test was used to compare the distributions of hyperthyroidism and euthyroidism after ATD withdrawal between the MLS and AFTN groups. The test showed no significant difference in thyroid function after ATD between both groups ( $p=$ 0.571). The median ATD treatment durations for the MLS and AFTN groups were 21.7 (range, 9.7-46.2) and 34.5 (range, 33.156.4) months, respectively. $\dagger$ Numbers in brackets indicate medians (ranges). ATD, antithyroid drug (methimazole or propylthiouracil); MLS, Marine-Lenhart syndrome; AFTN, autonomously functioning thyroid nodule.

Table 3 shows the distribution of thyroid function after RAI in the MLS and AFTN groups. Thyroid function was assessed on the last visit, 12-139.6 months after RAI administration. The rate of hypothyroidism after RAI was significantly higher in the MLS group than in the AFTN group (42.9\% [3/7] vs. 9.0\% [11/122], $p=0.005)$. The median dose of radioiodine-131 in both groups was 13 (interquartile range 13-13) $\mathrm{mCi}$.

\section{Discussion}

The original paper published by Marine and Lenhart in 1911 dealt with 8 patients in whom the thyroid pathology revealed the presence of adenoma with an iodine content lower than the level in the extranodular tissue [22]. Charkes [1] later reported, in 1972, ten patients with Graves' disease with incidental functioning nodules and named the disease as MLS. Chandramouly et al. [23] defined this syndrome as a variant of Graves' disease with TSH-dependent functional nodule(s), that is, poorly functioning nodule(s) that acquired functionality via TSH stimulation. Konno et al. [11] referred to this syndrome as the coexistence of Graves' disease and AFTN. Thereafter, the coexistence of thyroid functioning nodule(s) and Graves' disease has been called MLS. Thus, the definition and concept of this syndrome have changed with time: "TSH-dependent functional nodule(s)" $[8,24,25]$ and "coexistence of Graves' disease and AFTN" $[2,6,7,9,10,12-19,26-29]$. However, most of the previous reports emanated from studies conducted in iodine-defi-
Table 3. Comparison of thyroid functions after RAI between the MLS and AFTN groups

\begin{tabular}{llrc}
\hline & Hyperthyroidism & Euthyroidism & Hypothyroidism \\
\hline MLS $(n=7)$ & 0 & $4[57.1 \%]$ & $3[42.9 \%]$ \\
AFTN $(n=122)$ & $4[3.3 \%]$ & $107[87.7 \%]$ & $11[9.0 \%]$ \\
\hline
\end{tabular}

The median follow-up durations after RAI in the MLS and AFTN groups were 46.9 (range, 19.5-140) and 44.6 (range, 12.0134) months, respectively. Pearson's $\chi^{2}$ test was used to compare thyroid functions after RAI between the MLS and AFTN groups. The test showed a significant difference in thyroid function after RAI between both groups $(p=0.019)$. Data indicate the numbers of patients (proportion in the group). MLS, Marine-Lenhart syndrome; AFTN, autonomously functioning thyroid nodule; $\mathrm{RAI}$, radioactive iodine.

cient areas. Here, to investigate the clinical significance of the current definition of MLS, we analysed the frequency, treatment, and prognosis of the syndrome at a hospital that specializes in diseases of the thyroid in Japan, an iodinesufficient country.

Although $0.8-4.3 \%$ of patients with Graves' disease were reported to have TSH-dependent functional nodule(s) $[1,8,30]$, the prevalence of AFTN in those with Graves' disease has not been reported. Sasaki et al. [6], who included only a small number of patients in their study, suggested that $0.42 \%$ of patients with Graves' disease had AFTN. Here, we systematically analysed patients with thyrotoxicosis and coexisting nodules and showed that the prevalence of the syndrome was $0.26 \%$ of patients with Graves' disease. Thus, the prevalence of MLS in Japan is assumed to be $0.26-0.42 \%$ of all Graves' disease. The proportion of patients with MLS to those with thyrotoxicosis with nodule(s) was $1.8 \%$ and that of MLS to Graves' disease with nodule(s) was 3.8\%.

The frequency of AFTN is low in iodine-sufficient areas but high with about half the degree of the frequency of Graves' disease in the presence of the nodules. Several studies identified that $30-50 \%$ of AFTN cases are associated with normal TSH levels [31-33]. Belfiore et al. [34] reported the correlation between nodule size and prevalence of thyrotoxicosis among 740 patients with AFTNs in an iodine-sufficient area. Thyrotoxicosis was present in $43.3 \%$ of nodules larger than $30 \mathrm{~mm}$, whereas it was present in $10.0 \%$ of nodules smaller than $25 \mathrm{~mm}$. Thus, TSH suppression tended to occur when the nodule exceeded $30 \mathrm{~mm}$. In this 
study, the median diameter of nodules in the MLS group was $27 \mathrm{~mm}$ (less than $30 \mathrm{~mm}$ ) and significantly smaller than that in the AFTN group. Among cases with small nodules, AFTNs might not suppress TSH, whereas MLS cases presented with overt hyperthyroidism due to Graves' disease.

TRAb may be positive in some patients with AFTNs [35]. Differential diagnosis between these patients and those with MLS is based on thyroid scan findings. In patients with AFTNs, increased tracer uptake is seen in the areas of nodule(s) identified on ultrasonography, and the rest of the thyroid gland is suppressed. In contrast, in MLS, the area of the nodule(s) is particularly intense, with diffuse uptake in the thyroid gland [12]. No TRAb-positive AFTN was detected in this study.

In this study, the median age was 41 (range 5-94) years in the Graves' disease group and 53 (range 13-89) years in the AFTN group, and women comprised 81.0 and $90.8 \%$ in each group, respectively, which were close to the previous reports in Japan [36-40]. Therefore, the population of patients with hyperthyroidism in this study is considered representative of the Japanese population. We found that there was no difference in the clinical course after ATD withdrawal between the MLS and AFTN groups. Regarding RAI therapy, interestingly, more patients with MLS than those with AFTNs became hypothyroid despite similar nodular sizes and RAI doses. It is reasonable that RAI is trapped in the tissue of patients with Graves' disease in addition to functional nodules in MLS under suppression with serum TSH. Assuming that is the case, RAI therapy could be a good choice of treatment for MLS. As inducing hypothyroidism is a treatment goal among patients with Graves' disease, Tx including the nodule(s) is another choice of treatment for MLS.

One limitation of this study is that the timing of thyroid function evaluation after RAI was different in each case. Another limitation is that it is difficult to compare the frequency of thyroid nodules in this study with previous reports because of the improved diagnostic accuracy of recent ultrasonography. Lastly, some cases of Graves' disease and AFTNs were difficult to diagnose. "Undetermined thyrotoxicosis" would include "TRAb-negative Graves' disease" and "AFTN without sufficient accumulation on scintigraphy."

In conclusion, the rate of MLS among patients with Graves' disease was $0.26 \%$ in Japan. Although this syndrome is treated in accordance with AFTNs, RAI therapy induces hypothyroidism more frequently than in patients with AFTNs probably because RAI is taken up in the surrounding Graves' tissues.

\section{Acknowledgement}

We thank Ms. Izumi Otsuka for extracting the data from the electronic medical chart system.

\section{Statement of Ethics}

The protocol was approved by the Ethics Committee of Kuma Hospital (approval number: 20200213-6). Informed consent was obtained from each patient prior to the initiation of this study.

\section{Conflict of Interest Statement}

The authors have no conflicts of interest to declare.

\section{Funding Sources}

This research received no grant funding.

\section{Author Contributions}

All authors contributed to the study design and discussed the results and conclusions of this work. H.D. collected and analysed the data and wrote the manuscript with support from M.N. E.N. conceived the original idea and supervised the project.

\section{References}

1 Charkes ND. Graves' disease with functioning nodules (Marine-Lenhart syndrome). J Nucl Med. 1972 Dec;13(12):885-92.

2 Sharma A. Marine-Lenhart syndrome in two adolescents, including one with thyroid cancer: a case series and review of the literature. J Pediatr Endocrinol Metab. 2017 Nov 27; 30(12):1237-43.
3 DeGroot LJ, Larsen PR, Hennemann G. Graves' disease and the manifestations of thyrotoxicosis. In: The thyroid and its diseases. 6th ed. New York: Churchill Livingstone; 1996. p. 372.

4 Biersack HJ, Biermann K. The Marine-Lenhart syndrome revisited. Wien Klin Wochenschr. 2011 Aug;123(15-16):459-62.
5 Paschke R. Toxic adenoma and toxic multinodular goiter. In: Braverman LE, Cooper DS, editors. Werner \& Ingbar's the thyroid: a fundamental and clinical text. 10th ed. Philadelphia: Wolters Kluwer Williams \& Wilkins; 2013. p. 400.

6 Sasaki J. TSH-hypersensitive thyroid may cause hyperthyroidism (a hypothesis). Official J Jpn Assoc Endocr Surgeons Jpn Soc Thyroid Surg. 2014;31:303-9. 
7 Waldherr C, Otte A, Haldemann A, MüllerBrand J. [Marine-Lenhart syndrome: a case observation upon 18 years]. Nuklearmedizin. 1999;38(8):345-8.

8 Carnell NE, Valente WA. Thyroid nodules in Graves' disease: classification, characterization, and response to treatment. Thyroid. 1998 Aug;8(8):647-52.

9 Chatzopoulos D, Iakovou I, Moralidis E. Marine-Lenhart syndrome and radioiodine-131 treatment. Thyroid. 2007 Apr;17(4):373-4.

10 Avci E, Narci H. Coexistence of Graves' disease and toxic adenoma: a rare presentation of Marine-Lenhart syndrome. J Ayub Med Coll Abbottabad. 2015 Jan-Mar;27(1):24850.

11 Konno N, Hirokawa J, Abe S. A case of Graves' disease associated with an autonomously functioning thyroid nodule (AFTN) (MarineLenhalt syndrome) which spontaneously became a cold nodule. Endocrinol Jpn. 1988 Oct:35(5):753-8.

12 Cakir M. Marine-Lenhart syndrome. J Natl Med Assoc. 2005 Jul;97(7):1036-8.

13 Uludag M, Aygun N, Ozel A, Yener Ozturk F, Karasu R, Ozguven BY, et al. A rare presentation of autonomously functioning papillary thyroid cancer: malignancy in marine-Lenhart syndrome nodule. Case Rep Surg. 2016; 2016:8740405.

14 Atmaca H, Çolak R, Yazici ZA, Kefeli M, Tosun FC. Marine-Lenhart syndrome with papillary thyroid carcinoma. J Res Med Sci. 2015 Apr;20(4):412-5.

15 Scherer T, Wohlschlaeger-Krenn E, BayerleEder M, Passler C, Reiner-Concin A, Krebs $\mathrm{M}$, et al. A case of simultaneous occurrence of Marine-Lenhart syndrome and a papillary thyroid microcarcinoma. BMC Endocr Disord. 2013 May 8;13:16.

16 Stanzel S, Gyürüs P, Kresnik E, Aigner R. A rare case of marine-Lenhart syndrome with unilateral endocrine orbitopathy and hürthle cell carcinoma. Nuklearmedizin. 2017 Aug $14 ; 56(4): \mathrm{N} 28-30$.

17 Nishikawa M, Yoshimura M, Yoshikawa N, Toyoda N, Yonemoto T, Ogawa Y, et al. Coexistence of an autonomously functioning thyroid nodule in a patient with Graves' disease: an unusual presentation of marine-Lenhart syndrome. Endocr J. 1997 Aug;44(4): $571-4$.

18 Lombardi M, Tonacchera M, Macchia E. A new case of marine-Lenhart syndrome with a papillary thyroid carcinoma. Clin Case Rep. 2018 Dec;6(12):2299-302.
19 Poertl S, Kirner J, Saller B, Mann K, Hoermann R. T3-release from autonomously functioning thyroid nodules in vitro. Exp Clin Endocrinol Diabetes. 1998;106(6):489-93.

20 Nagataki S. The average of dietary iodine intake due to the ingestion of seaweeds is 1.2 $\mathrm{mg} /$ day in Japan. Thyroid. 2008 Jun;18(6): 667-8.

21 Kahaly GJ, Bartalena L, Hegedüs L, Leenhardt L, Poppe K, Pearce SH. 2018 European Thyroid association guideline for the management of Graves' hyperthyroidism. Eur Thyroid J. 2018 Aug;7(4):167-86.

22 Marine D, Lenhart CH. Pathological anatomy of exophthalmic goiter. Arch Intern Med. 1911;VIII(3):265-316

23 Chandramouly B, Mann D, Cunningham RP, Giegerich E. Marine-Lenhart syndrome. Graves' disease with poorly functioning nodules. Clin Nucl Med. 1992 Nov;17(11):905-6.

24 Mohan JR, Santhosh S, Sood A, Bhattacharya A, Mittall BR. Pertechnetate thyroid scan in Marine-Lenhart syndrome. Indian J Nucl Med. 2013 Apr;28(2):125-6.

25 Harisankar CN, Preethi GR, Chungath BB. Hybrid SPECT/CT evaluation of MarineLenhart syndrome. Clin Nucl Med. 2013 Feb; 38(2):e89-90.

26 Neuman D, Kuker R, Vendrame F. MarineLenhart syndrome: case report, diagnosis, and management. Case Rep Endocrinol. 2018;2018:3268010.

27 Damle NA, Mishra R. Identifying marineLenhart syndrome on a (99m)Tc-pertechnetate thyroid scan. Indian J Endocrinol Metab. 2013 Mar; 17(2):366

28 Takei M, Ishii H, Sato Y, Komatsu M. A case of marine-Lenhart syndrome with a negative TSH receptor antibody titer successfully treated with a fixed, low dose of I (131). Case Rep Endocrinol. 2014;2014:423563.

29 Joven $\mathrm{MH}$, Anderson RJ. Marine-Lenhart syndrome. Endocrine. 2015 Jun;49(2):570-1.

30 Park H, Zieverink S, Ransburg R. Marine-lenhart syndrome (Graves' disease with poorly functioning nodules). Thyroid research VIII. Proceedings of the 8 th international thyroid congress. Canberra: Australian Academy of Science; 1980:641-4.

31 Treglia G, Trimboli P, Verburg FA, Luster M, Giovanella L. Prevalence of normal TSH value among patients with autonomously functioning thyroid nodule. Eur J Clin Invest. $2015 \mathrm{Jul}$; 45(7):739-44.
32 Ianni F, Perotti G, Prete A, Paragliola RM, Ricciato MP, Carrozza C, et al. Thyroid scintigraphy: an old tool is still the gold standard for an effective diagnosis of autonomously functioning thyroid nodules. J Endocrinol Invest. 2013 Apr;36(4):233-6.

33 Chami R, Moreno-Reyes R, Corvilain B. TSH measurement is not an appropriate screening test for autonomous functioning thyroid nodules: a retrospective study of 368 patients. Eur J Endocrinol. 2014 Apr;170(4):593-9.

34 Belfiore A, Sava L, Runello F, Tomaselli L, Vigneri R. Solitary autonomously functioning thyroid nodules and iodine deficiency. J Clin Endocrinol Metab. 1983 Feb;56(2):283-7.

35 Pedersen IB, Knudsen N, Perrild H, Ovesen L, Laurberg P. TSH-receptor antibody measurement for differentiation of hyperthyroidism into Graves' disease and multinodular toxic goitre: a comparison of two competitive binding assays. Clin Endocrinol. 2001 Sep;55(3): 381-90.

36 Hiraiwa T, Ito M, Imagawa A, Takamatsu J, Kuma K, Miyauchi A, et al. Restriction of dietary Iodine does not ameliorate the early effect of anti-thyroid drug therapy for Graves' disease in an area of excessive iodine intake. J Endocrinol Invest. 2006 Apr;29(4):380-4.

37 Konishi T, Okamoto Y, Ueda M, Fukuda Y, Harusato I, Tsukamoto Y, et al. Drug discontinuation after treatment with minimum maintenance dose of an antithyroid drug in Graves' disease: a retrospective study on effects of treatment duration with minimum maintenance dose on lasting remission. Endocr J. 2011;58(2):95-100.

38 Sato S, Noh JY, Sato S, Suzuki M, Yasuda S, Matsumoto $\mathrm{M}$, et al. Comparison of efficacy and adverse effects between methimazole 15 $\mathrm{mg}+$ inorganic iodine $38 \mathrm{mg} /$ day and methimazole $30 \mathrm{mg} /$ day as initial therapy for Graves' disease patients with moderate to severe hyperthyroidism. Thyroid. 2015 Jan; 25(1):43-50.

39 Yano Y, Sugino K, Akaishi J, Uruno T, Okuwa $\mathrm{K}$, Shibuya H, et al. Treatment of autonomously functioning thyroid nodules at a single institution: radioiodine therapy, surgery, and ethanol injection therapy. Ann Nucl Med. 2011 Dec;25(10):749-54.

40 Ikekubo K, Hino M, Ito H, Otani M, Yamaguchi H, Saiki Y, et al. Thyroid carcinoma in solitary hot thyroid lesions on Tc-99m sodium pertechnetate scans. Ann Nucl Med. 1989 Mar;3(1):31-6. 